\title{
Stereotactic Vacuum-Assisted Core Biopsy Results for Non- Palpable Breast Lesions
}

\author{
Filiz Agacayak ${ }^{1 *}$, Alper Ozturk ${ }^{2}$, Atilla Bozdogan ${ }^{3}$, Derya Selamoglu ${ }^{2}$, Gul Alco $^{4}$, \\ Cetin Ordu ${ }^{5}$, Kezban Nur Pilanci ${ }^{5}$, Refik Killi ${ }^{1}$, Vahit Ozmen ${ }^{2}$
}

\begin{abstract}
Background: The increase in breast cancer awareness and widespread use of mammographic screening has led to an increased detection of (non-palpable) breast cancers that cannot be discovered through physical examination. One of the methods used in the diagnosis of these cancers is vacuum-assisted core biopsy, which prevents a considerable number of patients from undergoing surgical procedures. The aim of this study was to present the results of stereotactic vacuum-assisted core biopsy for suspicious breast lesions. Materials and Methods: Files were retrospectively scanned and data on demographic, radiological and pathological findings were recorded for patients who underwent stereotactic vacuum-assisted core biopsy due to suspicious mammographic findings at the Interventional Radiology Centre of the Florence Nightingale Hospital between January 2010, and April 2013. Statistical analysis was carried out using Pearson's Chi-square, continuity correction, and Fisher's exact tests. Results: The mean age of the patients was 47 years (range: 36-70). Biopsies were performed due to BIRADS 3 lesions in 8 patients, BIRADS 4 lesions in 77 patients, and BIRADS 5 lesions in 3 patients. Mammography elucidated clusters of microcalcifications in 73 patients $(83 \%)$ and focal lesions (asymmetrical density, distortion) in 15 patients (17\%). In terms of complications, 1 patient had a hematoma, and 2 patients had ecchymoses $(3 / 88 ; 3.3 \%)$. The histopathologic results revealed benign lesions in 63 patients $(71.6 \%)$ and malignant lesions in 25 patients $(28.4 \%)$. The mean duration of the procedure was 37 minutes (range: 18-55). Although all of the BIRADS 3 lesions were benign, 22 (28.6\%) of the BIRADS 4 lesions and all of the BIRADS 5 lesions were malignant. Among the malignant cases, $80 \%$ were in situ, and $20 \%$ were invasive carcinomas. These patients underwent surgery. Conclusions: In cases where non-palpable breast lesions are considered to be suspicious in mammography scans, the vacuum-assisted core biopsy method provides an accurate histopathologic diagnosis thus preventing a significant number of patients undergoing unnecessary surgical procedures.
\end{abstract}

Keywords: Mammography - nonpalpable breast lesion - stereotactic vacuum - assisted core biopsy

Asian Pac J Cancer Prev, 15 (13), 5171-5174

\section{Introduction}

The current diagnostic approach to lesions detected in the breast involves the method of ultrasound-assisted biopsy. However, microcalcification clusters and parenchymal distortions detected in mammography cannot always be visualized in the ultrasound images. Therefore, a method of stereotactic vacuum-assisted core (SVC) biopsy has come into use for histopathologic diagnosis of these lesions (Tothova et al., 2013). Through the extensive use of mammography in screening for breast cancer, widespread national breast cancer screening programs, and the increase in breast cancer awareness, non-palpable suspicious lesions are being detected more frequently (NHS Breast Screening Programme, 2009; Dogan et al., 2012; Wang et al., 2012; Fouladi et al., 2013; Uyeturk et al., 2013; Avci et al., 2014). Until a few years ago, surgical methods were used for histopathologic evaluation of such lesions that were invisible under ultrasonography. However, a greater number of these lesions can now be accurately diagnosed through the modern stereotactic vacuum-assisted core biopsy method, and thus surgical interventions can be minimized and inaccurate diagnoses can largely be prevented (NHS Breast Screening Programme \& Association of Breast Surgery at BASO 2010). Further advantages of the SVC biopsy method in comparison with surgery are the short procedure duration, minimal invasiveness, and lower cost (Huang et al., 2014).

The aim of this study is to present the results of the SVC biopsy technique in cases of suspicious breast lesions detected through mammography, and to investigate success and efficacy of the method.

${ }^{1}$ Department of Radiology, ${ }^{2}$ Department of Breast Surgery, ${ }^{3}$ Department of Biostatistics, Istanbul Florence Nightingale Hospital, ${ }^{4}$ Department of Radiation Oncology, Gayrettepe Florence Nightingale Hospital, ${ }^{5}$ Department of Medical Oncology, Faculty of Medicine, Istanbul Bilim University, Istanbul, Turkey*For correspondence: filizagacayak@yahoo.com 


\section{Materials and Methods}

\section{Patient selection}

For the purposes of this study, we evaluated the demographic, radiologic and pathologic findings, and treatment data of patients who underwent stereotactic vacuum-assisted core biopsy procedures at the Interventional Radiology Centre of the Sisli Florence Nightingale Hospital, between January 2010, and April 2013.

\section{Procedural technique}

The patient's breast to undergo the procedure has been placed on a specially designed prone table. After the target breast was compressed, a lateral and craniocaudal images were made. With the help of these images, the $x-y$ coordinates of the lesion were determined and the stereotactic images were obtained using a $15^{\circ}$ angle bilaterally. The depth of the lesion was measured and the $\mathrm{z}$ coordinate was set. Facilitated by software, the biopsy insertion point was marked on the skin. After the local anesthesia was administered, a small incision was made with a scalpel. The biopsy needle was inserted through the incision and fired. In each patient, samples were taken by a minimum of 6 firings clockwise and counter-clockwise. When sampling was finished, the metal marker was positioned and the procedure was completed.

\section{Statistical analysis}

In the processing of the study results, statistical analyses were carried out using the SPSS (Statistical Package for Social Sciences) for Windows 17.0 software. During the evaluation of the study data, besides the descriptive statistical methods (mean, median, number and percentage), Pearson's Chi-Square, Continuity Correction, and Fisher's Exact Test methods were employed for the qualitative comparison of the groups. The results were evaluated within a $95 \%$ confidence interval and statistical significance was based on a value of $\mathrm{p}<0.05$.

\section{Results}

The mean age of 88 patients who were included in the study was 47 years (range: 36-70). Radiological examination before the procedure had elucidated microcalcifications in clusters in 73 patients $(83 \%)$ and focal lesions (asymmetrical density, distortion) in 15 patients $(17 \%)$. Sixty-eight patients $(77 \%)$ had a single focal point in one breast; the procedure was repeated in both breasts or on two focal points in one breast in 10 patients $(11.3 \%)$. After the procedure, 1 patient $(1.1 \%)$ had a hematoma, while 2 patients $(2.2 \%)$ had ecchymosis. The mean duration of the procedure was calculated as 37 minutes (range: 18-55). Histopathologic results revealed benign lesions in 63 patients $(71.6 \%)$ and malignant lesions in 25 patients (28.4\%). Among the malignant cases, $80 \%$ had ductal carcinoma in situ (DCIS), and $20 \%$ had invasive carcinomas (Table 1).

Although all of the BIRADS (Breast Imaging Reporting and Data System) 3 mammographic lesions ( $\mathrm{n}=8$ patients) were benign, $28.6 \%$ ( $\mathrm{n}=22$ patients) among
Table 1. Patient Characteristics $(\mathbf{n}=\mathbf{8 8})$

\begin{tabular}{lrrrr}
\hline Characteristics & $\mathrm{n}$ & $\%$ & Mean & Range \\
\hline Age & 88 & 100 & 47 & $36-70$ \\
Mammography result & & & & \\
$\quad$ Microcalcification & 73 & 83 & & \\
$\quad$ Focal lesion & 15 & 17 & & \\
BIRADS Classification & & & & \\
$\quad$ III & 8 & 9.1 & & \\
IV & 77 & 87.5 & & \\
V & 3 & 3.4 & 37 & $18-55$ \\
Duration of procedure (min) & 88 & 100 & & \\
Complications & & & & \\
$\quad$ Yes & 3 & 3.4 & & \\
$\quad$ No & 85 & 96.6 & & \\
Biopsy result & & & & \\
$\quad$ Malignant & 25 & 28.4 & & \\
$\quad$ Benign & 63 & 71.6 & & \\
Malignant tumour type & & & & \\
$\quad$ Ductal carcinoma in situ & 20 & 80 & & \\
$\quad$ Invasive carcinoma & 5 & 20 & & \\
Benign tumour type & & & \\
$\quad$ Atypical hyperplasia & 4 & 6.3 & \\
$\quad$ Other & 59 & 93.7 & \\
Surgery following biopsy & & & \\
$\quad$ Performed & 29 & 33 & \\
$\quad$ Decided to follow up & 59 & 67 & \\
\hline
\end{tabular}

Table 2. Histopathological Evaluation of Clinical Findings

\begin{tabular}{|c|c|c|c|c|c|}
\hline \multirow[b]{3}{*}{ Clinical findings } & \multicolumn{4}{|c|}{ Biopsy result } & \multirow[b]{3}{*}{$\mathrm{p}$} \\
\hline & \multicolumn{2}{|c|}{ Malignant } & \multicolumn{2}{|c|}{ Benign } & \\
\hline & $\mathrm{n}$ & $\%$ & $\mathrm{n}$ & $\%$ & \\
\hline BIRADS classification & & & & & $0.005^{*}$ \\
\hline III & 0 & 0 & 8 & 100 & \\
\hline IV & 22 & 28.6 & 55 & 71.4 & \\
\hline $\mathrm{V}$ & 3 & 100 & 0 & 0 & \\
\hline Mammography result & & & & & 0.541 \\
\hline Microcalcification & 22 & 30.1 & 51 & 69.9 & \\
\hline Focal lesion & 15 & 20 & 12 & 80 & \\
\hline
\end{tabular}

the BIRADS 4 lesions and all of the BIRADS 5 lesions $\left(\mathrm{n}=3\right.$ patients) were malignant $\left(\mathrm{X}^{2}=10.736 ; \mathrm{p}=0.005\right)$. Malignancies were detected in $30 \%$ among the 73 patients with microcalcifications and in $20 \%$ of the patients with focal lesions in the mammographic images $(p=0.541$; Table 2). Four patients whose vacuum-assisted core biopsies indicated atypical ductal hyperplasia underwent mammographic marking and excisions. No malignancies were detected histopathologically in samples from these patients.

\section{Discussion}

Stereotactic vacuum-assisted core biopsy (SVC) is being used with increasing frequency for the pathologic diagnosis of nonpalpable breast lesions that are invisible under USG or detected in the form of microcalcification clusters, asymmetrical increases in density, or spiculated masses in mammography (Tonegutti and Girardi 2008). Among the advantages of this method are its minimallyinvasive approach, uncomplicated application, quick results, low cost, reliability and enabling improvement 
in practice pattern of diagnostic tests (i.e. mammogram) (Domeyer et al., 2013; Tothova et al., 2013). Through the widespread promotion of national breast cancer screening programs and increase in breast cancer awareness, millions of women are having mammographies each year where a number of suspicious lesions are identified. Consequently, the number of SVC biopsies performed is increasing every day (NHS Breast Screening Programme 2009).

The diagnosis and treatment management of non-palpable suspicious lesions detected through mammography depends on the experience and skill of the team of surgeons, radiologists, and pathologists dealing with breast cancer (Perry et al., 2006). Studies have shown that false negative results after SVC vary between $0.45 \%$ and 22.2\% (Shah et al., 2003; Jackman et al., 2009). The cause of this difference is associated with the size of the biopsy needle. On the other hand, failure ratios of this procedure vary between $1.6 \%$ and $13 \%$ (Philpotts et al., 1997; Kettritz et al., 2004). Technological progress in the needle design and stereotactic techniques has led to the possibility of more accurate diagnoses. This high coherence between the pre- and postsurgical diagnoses minimizes surgery and avoids incorrect diagnoses (NHS Breast Screening Programme and Association of Breast Surgery at BASO 2010; Ames and Britton, 2011). Moreover, procedures were successfully performed in our study by an experienced breast radiologist, and pathology results were found to be in compliance with the radiological finding.

Leaving a radio-opaque marker in the sampling area following an SVC biopsy is of great importance. In cases where the lesion in the biopsy area has to be excised or another biopsy is required in the same area, the metal marker serves as a guide. The specimen radiogram taken after the biopsy shows the area where the SVC was performed and verifies the correct positioning of the biopsy needle (microcalcification, asymmetrical density, etc.). The occurrence rate for microcalcifications observed in specimen radiograms following SVC biopsies has been reported as 95-100\% (Meyer et al., 1999). In our study, specimen radiograms taken after SVC demonstrated that samples had been taken from the correct target points.

Patients who have undergone SVC biopsies have been evaluated in terms of malignancies. In the study by Kettritz et al, the ratio of benign/malignant lesions was 2.7, where $27 \%$ of the lesions were malignant and $73 \%$ were benign (Kettritz et al., 2004). The ratio of the malignant cases was lower in the study by Issam et al. (13.8\%), but was higher $(42 \%)$ in the study by Tonegutti et al. (Faour et al., 2008; Tonegutti and Girardi, 2008). The high ratio of malignancies in the latter study was associated with the selection of patients from high-risk groups. Other studies have also led to similar results (Brenner et al., 2001; Ciatto et al., 2006). In our study, $71.6 \%$ of the lesions in the patients who had undergone SVC biopsies were benign, and $28.4 \%$ were malignant. The benign/malignant ratio was 2.52 .

The BIRADS 3 patient group usually consists of overanxious individuals who ask for biopsies themselves because they have positive family histories of cancer; patients whose follow up causes concerns; and those who are recommended for biopsy by a clinician (Rotter et al., 2003). In these patients, the ratio of malignancies after SVC has been reported to be between $0 \%$ and $19 \%$ (Mendez et al., 2004; Kettritz et al., 2004; Tonegutti and Girardi 2008). The variation in malignancy rates of BIRADS 3 lesions can be associated with a subjective assessment of these lesions by a radiologist's lack of expertise in breast lesions, and highly variable image quality. Also in our study, the biopsy results each of the patients who were mammographically assessed as BIRADS 3 were benign and no further surgical intervention was required in these patients.

The ratio of malignancies varies in the pathology results of BIRADS 4 lesions after SVC biopsies between 4\% and 18\% (Liberman et al., 1998; Tate et al., 2001; Mendez et al., 2004). In the study by Tonegutti et al., the prevalence of malignancy among BIRADS 4a lesions was $14 \%$, and rose to $92 \%$ when the BIRADS $4 \mathrm{~b}$ and BIRADS 5 lesions were evaluated together (Tonegutti and Girardi 2008). In our study, the BIRADS 4 lesions were not classified as a, b, or $\mathrm{c}$ and this constitutes a limitation of the study. The ratio of malignancies in the BIRADS 4 group was $28.6 \%(22 / 77)$.

The prevalence of malignancies among the lesions radiologically classified as BIRADS 5 was $100 \%$ in the study by Tonegutti, $79 \%$ in the study by Mendez, $92 \%$ in Liberman's study and 90\% in Tate's study (Liberman et al., 1998; Tate et al., 2001; Mendez et al., 2003; Tonegutti and Girardi, 2008). In our study, the pathology results of all the lesions assessed as BIRADS 5 were malignant.

The patients whose SVC biopsy results indicated an atypical ductal hyperplasia in our study underwent surgical excisions, which also confirmed the diagnosis of atypical ductal hyperplasia. In the study by Faour, the pathology results of the surgical excision subsequent to an SVC biopsy, which had indicated atypical ductal hyperplasia, also confirmed the diagnosis of atypical ductal hyperplasia (Faour et al., 2008). Both in Faour's (2008) and our study, the postsurgical pathology results also confirmed the biopsy results of patients whose SVC biopsy results were malignant. These results can be explained due to the abundant material that is collected through vacuumbiopsy for the pathological evaluation. In some studies, the whole lesion was removed through SVC biopsy and no malignancies were observed in the surgically excised piece (Kumaroswamy et al., 2008).

The stereotactic vacuum-assisted core biopsy method is reported to have to a very low rate of complications. The complications observed in the reviewed studies include ecchymoses, pain, hematoma, and formation of abscesses. The ratio of abscesses or hematoma necessitating surgical drainage seems to be around 0.1\% (Burbank et al., 1996). Although tumour seeding after SVC biopsy has been reported in some experimental studies, this has not been regarded as clinically relevant. No recurrence in the biopsy line or new tumoral tissue formation in the biopsied breast has been observed due to tumour seeding as a direct result of the biopsy (Liberman et al., 1999). In a study by Faour et al. (2008), 2\% of the patients had ecchymoses and another $2 \%$ were observed to develop hematoma; the ecchymoses healed without treatment and the hematomas 


\section{Filiz, Agacayak et al}

were controlled through local compression. In a study by Tonegutti and Girardi. (2008), only one patient had a large abscess as a serious complication and this patient was reported be an elderly individual with diabetes. Kettritz et al. (2004) reported that the complication rate was $1.4 \%$ and these included vasovagal syncope during procedure, hematoma formation, and inflammation in the biopsy area. Only $0.1 \%$ of these patients required surgical intervention. In our study, one patient $(1.1 \%)$ had a hematoma, and two patients $(2.2 \%)$ had ecchymoses. The patients who had developed ecchymoses recovered without treatment and the enlargement of the hematoma in one patient was controlled through compression.

Current studies have demonstrated that SVC biopsy is a reliable method for the pathological diagnosis of the non-palpable breast lesions that cannot be observed under USG. The sensitivity and specificity of SVC biopsy in these types of lesions make it a reliable alternative to excisional biopsy in diagnosis and planning of further definitive treatment. Although it is an efficient method for the pathological evaluation of BIRADS 3-5 lesions, our results indicate that a more selective approach should be adopted with BIRADS 3 lesions. In terms of cost effectiveness, the method brings advantages when compared with surgery because it is a well-tolerated method and reduces time off work (Lee et al., 2013).

In conclusion; stereotactic vacuum-assisted core breast biopsy performed on a prone table is a reliable and highly reproducible method, which is comfortable for patients, requires no hospitalization, and could replace diagnostic surgical biopsies.

\section{Acknowledgements}

The authors would like to thank Mr. David Chapman for his editing of the manuscript.

\section{References}

Ames V, Britton PD (2011). Stereotactically guided breast biopsy: a review. Insights Imaging, 2, 171-6.

Avci IA, Kumcagiz H, Altinel B, Caloglu A (2014). Turkish female academician self-esteem and health beliefs for breast cancer screening. Asian Pac J Cancer Prev, 15, 155-60.

Brenner RJ, Bassett LW, Fajardo LL, et al (2001). Stereotactic core-needle breast biopsy: a multi-institutional prospective trial. Radiology, 218, 866-72.

Burbank F, Parker SH, Fogarty TJ (1996). Stereotactic breast biopsy: improved tissue harvesting with the Mammotome. Am Surg, 62, 738-44.

Ciatto S, Houssami N, Ambrogetti D, et al (2007). Accuracy and underestimation of malignancy of breast core needle biopsy: the Florence experience of over 4000 consecutive biopsies. Breast Cancer Res Treat, 101, 291-97.

Dogan L, Gulcelik MA, Yuksel M, Uyar O, Reis E (2012). Wireguided localization biopsy to determine surgical margin status in patients with non-palpable suspicious breast lesions. Asian Pac J Cancer Prev, 13, 4989-92.

Domeyer PJ, Sergentanis TN, Katsari V, et al (2013). Screening in the era of economic crisis: Misperceptions and misuse from a longitudinal study on Greek women undergoing benign vacuumassisted breast biopsy. Asian Pac J Cancer Prev, 14, 5023-9.

Faour I, Al-Salam S, El-Terifi H, El Taji H (2008). The use of a vacuum-assisted biopsy device (Mammotome) in the early detection of breast cancer in the United Arab Emirates. Ann $N$
$Y$ Acad Sci, 1138, 108-13.

Fouladi N, Pourfarzi F, Mazaheri E, et al (2013). Beliefs and behaviors of breast cancer screening in women referring to health care centers in northwest Iran according to the Champion health belief model scale. Asian Pac J Cancer Prev, 14, 6857-62.

Huang M, Adrada BE, Candelaria R, et al (2014). Stereotactic breast biopsy: pitfalls and pearls. Tech Vasc Interv Radiol, 17, 32-9.

Jackman RJ, Marzoni FA Jr, Rosenberg J (2009). False-negative diagnoses at stereotactic vacuum-assisted needle breast biopsy: long-term follow-up of 1,280 lesions and review of the literature. AJR Am J Roentgenol, 192, 341-51.

Kettritz U, Rotter K, Schreer I, et al (2004). Stereotactic vacuumassisted breast biopsy in 2874 patients: a multicenter study. Cancer, 100, 245-51.

Kumaroswamy V, Liston J, Shaaban AM (2008). Vacuum assisted stereotactic guided mammotome biopsies in the management of screen detected microcalcifications: experience of a large breast screening centre. J Clin Pathol, 61, 766-9.

Lee KE, Kim HH, Shin HJ, Cha JH (2013). Stereotactic biopsy of the breast using a decubitus table: comparison of histologic underestimation rates between 11- and 8- gauge vacuum-assisted breast biopsy. Springerplus, $2,551$.

Liberman L, Abramson AF, Squires FB, et al (1998). The breast imaging reporting and data system: positive predictive value of mammographic features and final assessment categories. Am J Roentgenol, 171, 35-40.

Liberman L, Vuolo M, Dershaw DD, et al (1999). Epithelial displacement after stereotactic 11-gauge directional vacuumassisted breast biopsy. AJR Am J Roentgenol, 172, 677-81 .

Mendez A, Cabanillas F, Echenique M, et al (2004). Mammographic features and correlation with biopsy findings using 11-gauge stereotactic vacuum-assisted breast biopsy (SVABB). Ann Oncol, 14, 450-4.

Meyer JE, Smith DN, Lester SC, et al (1999). Large-core needle biopsy of nonpalpable breast lesions. JAMA, 281, 1638-41.

NHS Breast Screening Programme (2009) NHS breast screening programme review ISBN 978184463064 6. www. cancerscreening.nhs.uk//breastscreen/publications/2009review. html

NHS Breast Screening Programme and Association of Breast Surgery at BASO (2010) An audit of screen-detected breast cancers for the year of screening April 2008 to March 2009.

Perry N, Broeders M, de Wolf C, et al (2006). European guidelines for quality assurance in breast cancer screening and diagnosis, 4th edn, ISBN 92-79-01258-4.

Philpotts LE, Lee CH, Horvath LJ, Tocino I (1997) Canceled stereotactic core-needle biopsy of the breast: analysis of 89 cases, Radiology, 205, 423-8.

Rotter K, Haentschel G, Koethe D, et al (2003). Evaluation of mammographic and clinical follow-up after 755 stereotactic vacuum-assisted breast biopsies. Am J Surg, 186, 134-42.

Shah VI, Raju U, Chitale D, et al (2003). False-negative core needle biopsies of the breast: an analysis of clinic, radiologic, and pathologic findings in 27 consecutive cases of missed breast cancer. Cancer, 97, 1824-31.

Tate PS, Rogers EL, McGee EM, et al (2001). Stereotactic breast biopsy: a six year surgical experience. J Ky Med Assoc, 99, 98-103.

Tonegutti M, Girardi V (2008). Stereotactic vacuum-assisted breast biopsy in 268 nonpalpable lesions. Radiol Med, 113, 65-75.

Tothova L, Rauova K, Valkovic L, Vanovcanova L, Lehotska V (2013). Stereotactic vacuum-assisted breast biopsy: our experience and comparison with stereotactic automated needle biopsy. Bratisl Lek Listy, 114, 71-7.

Uyeturk U, Tatli AM, Gucuk S, et al (2013). Risk factors for stage IV breast cancer at the time of presentation in Turkey. Asian Pac J Cancer Prev, 14, 7445-49.

Wang XW, Xiong YH, Zen XQ, Lin HB, Liu QY. (2012). Diagnostic accuracy of ultrasonography guided fine-needle aspiration cytologic in staging of axillary lymph node metastasis in breast cancer patients: a meta-analysis. Asian Pac J Cancer Prev, 13, 5517-23. 